\title{
Neutron Transmission Spectrum of Liquid Lead Bismuth Eutectic
}

Yojiro Oba ${ }^{1, a^{*}}$, Daisuke Ito ${ }^{2, b}$, Yasushi Saito ${ }^{2}$, Yohei Onodera ${ }^{2}$, Joseph Don Parker ${ }^{3}$, Takenao Shinohara ${ }^{4}$, and Kenichi Oikawa ${ }^{4}$

${ }^{1}$ Materials Sciences Research Center, Japan Atomic Energy Agency, 2-4, Shirakata, Tokai, Ibaraki, Japan

${ }^{2}$ Institute for Integrated Radiation and Nuclear Science, Kyoto University, 2, Asashiro-nishi, Kumatori, Osaka, Japan

${ }^{3}$ Comprehensive Research Organization for Science and Society, 162-1, Shirakata, Tokai, Ibaraki, Japan

${ }^{4}$ J-PARC Center, Japan Atomic Energy Agency, 2-4, Shirakata, Tokai, Ibaraki, Japan

a ohba.yojiro@jaea.go.jp, bitod@rri.kyoto-u.ac.jp

Keywords: Neutron Transmission Imaging, Pulsed Neutron, Lead Bismuth Eutectic

Abstract. Energy-resolved neutron transmission imaging experiment was carried out to characterize the liquid phase of lead bismuth eutectic (LBE). The neutron transmission image confirmed that the LBE is homogeneous in a sample vessel. The obtained neutron transmission spectrum of the liquid LBE shows a wavy behavior in the wavelength dependence. This behavior is attributed to neutron attenuation by the neutron diffraction of the liquid LBE, which can give information about the atomic structure of the liquid LBE similar to Bragg edge transmission in the crystalline solid phases.

\section{Introduction}

Lead bismuth eutectic (LBE, 44.5 mass\% Pb 55.5 mass\% Bi) is a candidate material of coolant for LBE-cooled accelerator driven systems (ADS) and fast breeder reactor (FBR) [1,2]. Understanding of thermal properties and flow behavior of the LBE are crucial for design and safety analysis of such ADS and FBR facilities. To characterize those properties of the LBE, structural information plays a key role because it is highly related to those properties. Recently, Ito et al. have clearly demonstrated that an energy-resolved neutron transmission imaging experiment is useful to study the structure of the LBE [3]. The analysis of Bragg edge transmission, which results from the neutron attenuation by neutron diffraction, provides the structural information about crystalline solid phases of the LBE [4].

However, the structures of liquid phases in the LBE were not considered in the previous study even though they are closely linked to the flow behavior in operation as the coolant. If the neutron diffraction contributions caused by the liquid phases of the LBE can be detected in the neutron transmission spectra, they probably give beneficial information about the atomic structures in the liquid phases in the same manner as the Bragg edge transmission. Therefore, we performed the energy-resolved neutron imaging measurements of the liquid phase of the LBE to observe the neutron diffraction contribution of the liquid LBE in the neutron transmission spectra.

\section{Experiment}

The experiments were carried out at the energy-resolved neutron imaging instrument BL22 RADEN and at the neutron beamline for observation and research use BL10 NOBORU in J- 
PARC [5,6]. The LBE was sealed in a rectangular sample vessel made of austenitic stainlesssteel plates with the thickness of $1 \mathrm{~mm}$. The thickness of the LBE was $10 \mathrm{~mm}$. The sample vessel was then covered with glass wool and aluminum tape for thermal insulation. The temperature of the LBE was measured using five thermocouples and controlled using cartridge heaters and cooling fans. Details are described elsewhere [3]. A neutron gas electron multiplier (nGEM) detector was used to obtain the neutron transmission images. The dimensions of each pixel and the effective area of the detector were $0.8 \times 0.8 \mathrm{~mm}$ and $100 \times 100 \mathrm{~mm}$, respectively. The sample was placed just before the detector.

\section{Results and discussion}

Fig. 1 (a) shows the neutron transmission image of the LBE in the sample vessel at $220{ }^{\circ} \mathrm{C}$ integrated between the wavelength of 0.1 and $0.6 \mathrm{~nm}$. Surface of the LBE is observed at 105 pixels $=84 \mathrm{~mm}$ from the bottom of the vessel (shown by a white broken line). In the neutron transmission image, five bar-shaped shadows extended from the side vessel walls are neutron attenuation by the thermocouples. Other than these features, the neutron transmission image shows horizontal stripe-like inhomogeneity. This is not originated by the LBE, but by the sample vessel. Fig. 1 (b) shows the neutron transmission image of the identical sample vessel after removing the LBE. The same horizontal stripe-like inhomogeneity is observed in the image. Since the neutron transmission image of only the sample vessel without the thermal insulator, i.e., only the stainless steel, exhibits flat neutron attenuation [Fig. 1 (c)], this inhomogeneity results from the glass wool and aluminum tape horizontally wound around the vessel.

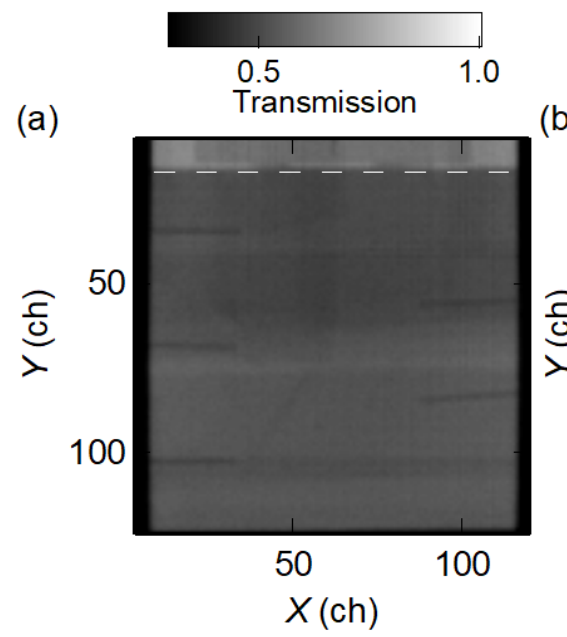

(b)

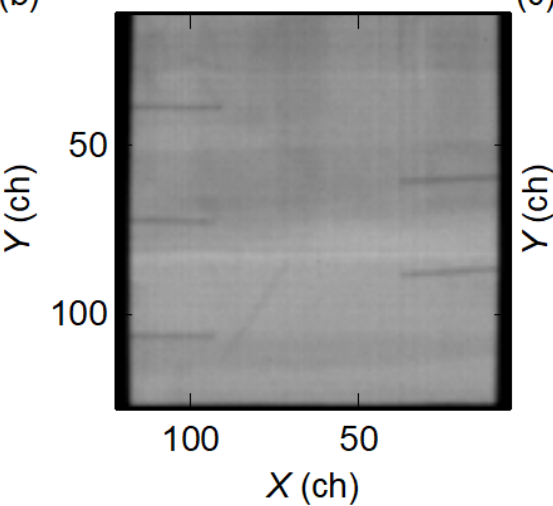

(c)

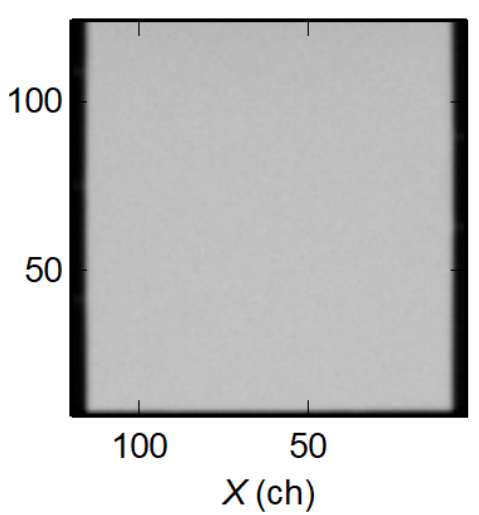

Fig. 1 Neutron transmission images integrated between wavelength of 0.1 and $0.6 \mathrm{~nm}$ of (a) LBE in sample vessel, (b) sample vessel after removing LBE, and (c) stainless steel plates of sample vessel. Horizontal white broken line indicates the surface of LBE. Black edge regions correspond to vessel wall made of stainless steel plates.

Based on the Beer-Lambert law [7], the neutron attenuation contribution of the sample vessel can be removed by dividing the neutron transmission of the LBE in the sample vessel by that of the empty vessel. Fig. 2 shows the divided neutron transmission image, which should be composed of only the LBE contribution. The horizontal stripe-like inhomogeneity disappears in the divided image. However, the white and black shadows of the thermocouples remain. This is probably caused by the moving of the thermocouples during the removal of the LBE from the sample vessel. Hereafter, only center parts of the neutron transmission images (shown by a white 
broken square in Fig. 2) are discussed to avoid unexpected effects of the thermocouples. The divided image of the LBE shows uniform neutron attenuation in the center parts. This reflects the uniformity of the LBE in the sample vessel.

Fig. 3 indicates the neutron transmission spectra averaged in the center parts of the neutron transmission images of the LBE in the sample vessel, the sample vessel after removing the LBE, and the sample vessel without the thermal insulator (= the austenitic stainless steel). The vertical dotted line indicates the Bragg cutoff of the austenitic stainless steel, where the wavelength is equal to twice the largest lattice spacing $2 d_{\max }$ in corresponding crystal structure. Below the



Fig. 2 Divided neutron transmission image of LBE. The white broken square is the region to calculate average neutron transmission. Bragg cutoff, all the neutron transmission spectra show the Bragg edge transmission from the austenitic stainless steel, while no Bragg edge transmission from the crystalline LBE is observed in the spectrum of the LBE. This indicates that the LBE is completely in the liquid phase. At the wavelength longer than the Bragg cutoff, although the Bragg edge transmission of the austenitic stainless steel no longer occurs, only the spectrum of the LBE curves gently. This is probably due to the liquid LBE.

To characterize the liquid LBE, the neutron transmission spectra was separated into the contributions of the liquid LBE, the austenitic stainless steel (= the sample vessel without the thermal insulator), and the thermal insulator. Dividing the neutron transmission of the LBE in the sample vessel by that of the empty sample vessel gives the contribution of the liquid LBE.

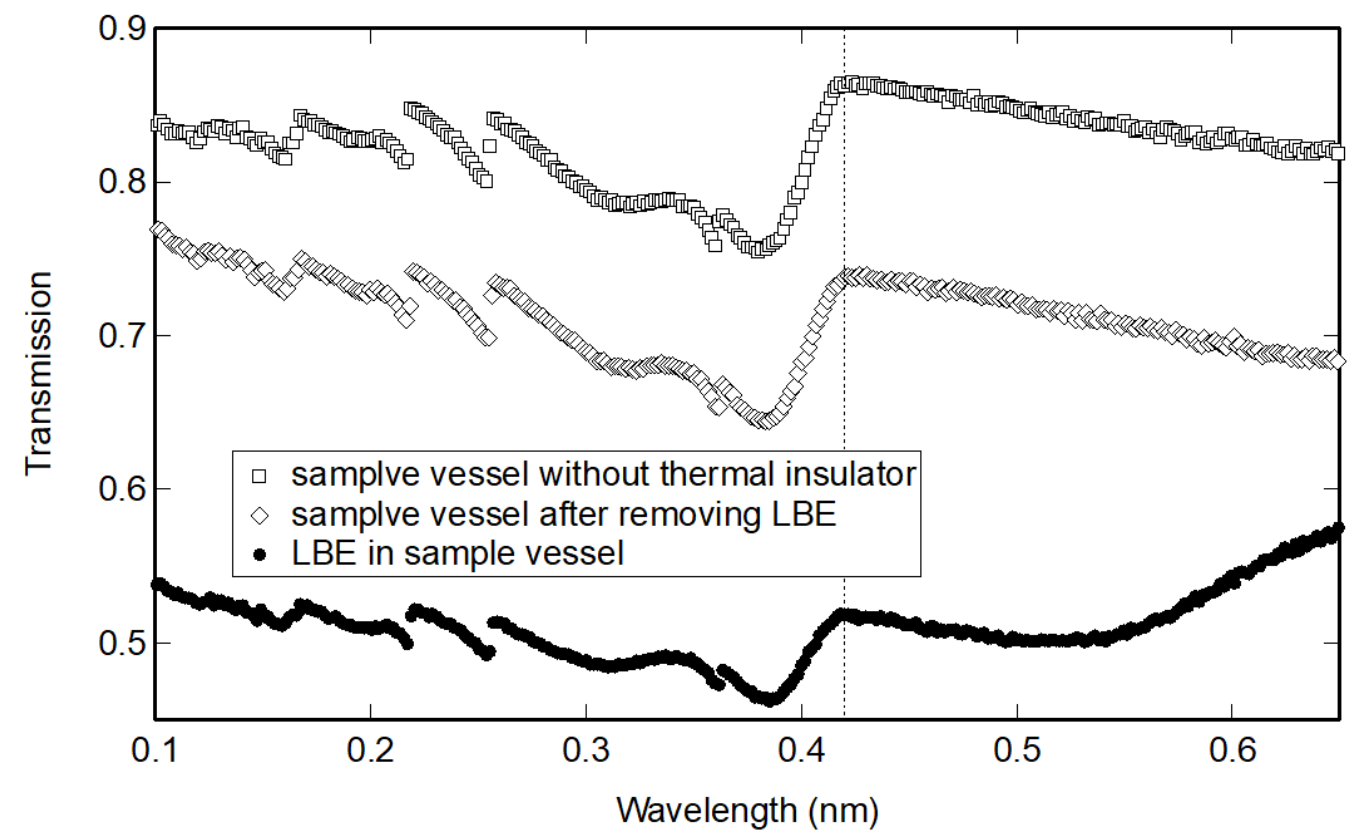

Fig. 3 Neutron transmission spectra. Black circles, open diamonds, and open squares denote LBE in sample vessel, sample vessel after removing LBE, and sample vessel withou thermal insulator. Vertical dotted line is Bragg cut-off of austenitic steel. 
Similarly, the contribution of the thermal insulator can be obtained by dividing the neutron transmission of the empty sample vessel by that of the austenitic stainless steel. Fig. 4 shows these contributions of the liquid LBE, the austenitic stainless steel, and the thermal insulator in the neutron transmission spectra. The Bragg edge transmission occurs merely in the contribution of the austenitic stainless steel. The contribution of the thermal insulator is featureless and almost monotonically decreases with increasing the wavelength. In contrast, the contribution of the liquid LBE has obviously different features. In addition to the gentle curve observed in Fig. 3, whole the spectrum shows a wavy behavior. Fig. 4 also shows an absorption contribution calculated from the chemical composition and the density of the LBE using the database of total cross-sections [1,7]. Since the absorption contribution is negligibly small, the wavy behavior of the liquid LBE is attributed to the attenuation by the neutron diffraction, which reflects the atomic structure of the liquid LBE. Therefore, the homogeneous neutron transmission image in Fig. 2 means that the atomic structure of the liquid LBE is uniform.

A recent study has demonstrated that the neutron attenuation coefficient is proportional to the scattering intensity integrated in all solid angles [8]. Although this study discussed only a contribution of small-angle neutron scattering (SANS), this idea can be simply expanded to neutron diffraction. Hence, broadened diffraction pattern of the liquid LBE [9] probably brings about the wavy behavior instead of the sharp Bragg edge transmission, which is connected with sharp Bragg peaks in the crystalline solid phases.

Based on the ref. [8], conventional analytical techniques for the neutron diffraction of the liquid phases can be also applied to the neutron transmission spectra. Such application enables to obtain the structural information about the liquid phases from the neutron transmission images. This will be useful to study the liquid LBE in flow channels. However, contributions of background, such as the sample vessel, have to be precisely removed to obtain meaningful information from the neutron transmission spectra.

\section{Summary}

In this study, the energy-resolved neutron transmission imaging experiment of the liquid LBE

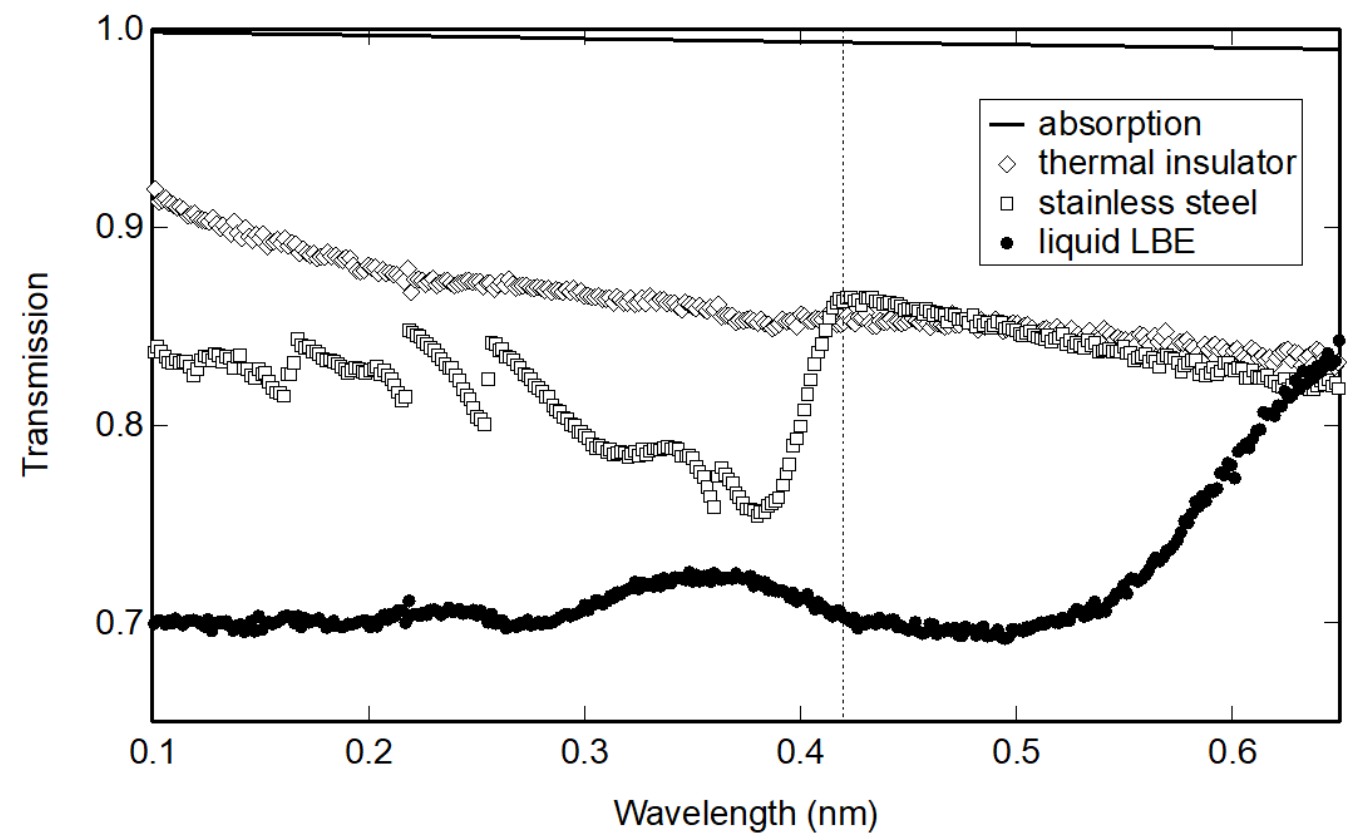

Fig. 4 Contributions of liquid LBE, stainless steel, and thermal insulator in the neutron transmission spectra. Black circles, open squares, and open diamonds denote contributions of liquid LBE, stainless steel, and thermal insulator, respectively. Solid line is calculated absorption contribution. 
was performed. The neutron transmission image shows the uniformity of the liquid LBE. The neutron transmission spectrum of the liquid LBE was successfully extracted from the neutron transmission spectra of the LBE in the sample vessel and the empty sample vessel. The wavy behavior of the neutron transmission spectrum of the liquid LBE is probably resulted from the broadened diffraction of the liquid LBE.

\section{Acknowledgement}

Neutron experiments at the MLF J-PARC were performed under Proposal Nos. 2016A0272, 2017A0144, and 2017B0120.

\section{References}

[1] C. Fazio, et al., Handbook on Lead-bismuth Eutectic Alloy and Lead Properties, Materials Compatibility, Thermal-hydraulics and Technologies 2015 Edition, OECD, 2015.

[2] V. Sobolev, Properties of Liquid Metal Coolants, in: R. J. M. Konings (Ed.), Comprehensive Nuclear Materials, Elsevier, Amsterdam, 2012, pp. 373-392. https://doi.org/10.1016/B978-0-08056033-5.00130-0

[3] D. Ito, Y. Saito, H. Sato, T. Shinohara, Visualiz ation of solidification process in leadbismuth eutectic, Phys. Proc. 88 (2017) 58-63. https://doi.org/10.1016/j.phpro.2017.06.007

[4] O. Takada, T. Kamiyama, Y. Kiyanagi, Study on phase transition of Pb-Bi eutectic alloy by neutron transmission spectroscopy, J. Nucl. Mater. 398 (2010) 129-131.

https://doi.org/10.1016/j.jnucmat.2009.10.022

[5] T. Shinohara, T. Kai, K. Oikawa, M. Segawa, M. Harada, T. Nakatani, M. Ooi, K. Aizawa, H. Sato, T. Kamiyama, H. Yokota, T. Sera, K. Mochiki, Y. Kiyanagi, Final design of the EnergyResolved Neutron Imaging System “RADEN” at J-PARC, J. Phys.: Conf. Ser. 746 (2016) 012007. https://doi.org/10.1088/1742-6596/746/1/012007

[6] K. Oikawa, F. Maekawa, M. Harada, T. Kai, S. Meigo, Y. Kasugai, M. Ooi, K. Sakai, M. Teshigawara, S. Hasegawa, M. Futakawa, N. Watanabe, Design and application of NOBORUNeutrOn Beam line for Observation and Research Use at J-PARC, Nucl. Instrum. Met. Phys. Res. A 589 (2008) 310-317. https://doi.org/10.1016/j.nima.2008.02.019

[7] A.-J. Dianoux, G. Lander, Neutron Data Booklet, Old City Publishing, Philadelphia, 2003.

[8] Y. Oba, S. Morooka, K. Ohishi, J. Suzuki, S. Takata, N. Sato, R. Inoue, T. Tsuchiyama, E. P. Gilbert, M. Sugiyama, Energy-resolved small-angle neutron scattering from steel, J. Appl. Cryst. 50 (2017) 334-339. https://doi.org/10.1107/S1600576717000279

[9] I. Kaban, W. Hoyer, Y. Plevachuk, V. Sklyarchuk, Atomic structure and physical properties of liquid Pb-Bi alloys, J. Phys.: Condens. Matter 16 (2004) 6335-6341. https://doi.org/10.1088/0953-8984/16/36/001 